\title{
TIME-VARYING LINEAR OBSERVER FOR TORQUE BALANCING ON A DI ENGINE
}

\author{
Jonathan Chauvin* Gille Corde ** Philippe Moulin ** \\ Michel Castagné ${ }^{* *}$ Nicolas Petit* Pierre Rouchon*
* Centre Automatique et Systèmes, École des Mines de Paris, 60, bd St Michel, 75272 Paris, France
** Institut Français du Pétrole, 1 et 4 Avenue de Bois Préau, 92852 Rueil Malmaison, France

\begin{abstract}
Torque balancing for DI engines aims at eliminating the vibration mode and decreasing the level of emissions induced by disturbances such as variability in injectors efficiency, inertia and geometry between cylinders. The contribution of this article is to present a real-time experimental setup along with simulations and experimental results of estimation of the indicated torque of a DI engine. We build a time-varying linear model of the system and design a linear observer to estimate the indicated torque produced by each cylinder. This is achieved with the instantaneous engine speed as only measurement. This work can be used in a next step when unbalance could be corrected in an adaptive manner by controlling the mass injected into each cylinder.
\end{abstract}

Keywords: Time-varying Control, Observer Design, Engine Modelling, Torque Balancing, Real-time

\section{INTRODUCTION}

Torque balancing for diesel engines is a major feature of modern engine controllers for two main reasons:

- Diesel injection systems introduce a bias in injected masses between cylinders (relative errors can be up to $20 \%$ at low engine speed). Torque production varies from cylinder to cylinder in a monotonic way with respect to the injected fuel mass.

- This torque variation is a periodic time function and generates a undesired vibration mode in the engine.

This phenomenon is important at low engine speed and idle mode conditions. To estimate torque production from each cylinder we use the instantaneous engine speed at the crankshaft. Currently, an engine speed measurement every $45^{\circ}$ of crankshaft rotation seem sufficient to estimate torque balance and to correct it in an adaptive manner by controlling the mass injected in each cylinder.

Torque balance determination by the measurement of the crankshaft angle has been addressed previously in the literature. Most of the solutions proposed have their foundation on a Direct or Indirect Fourier Transform as in (Jianqiu et al., 2002a), (Jianqiu et al., 2002b) and (Ginoux and Champoussin, 1997) or are founded on the derived measures from crankshaft position (van Nieuwstadt and Kolmanovsky, 1997). Our approach use a time-varying model for the design of an engine torque observer.

The goal of our work is to build a more accurate observer of torque balancing in transient mode (according to torque and engine speed). The paper organizes as follows. In the Section 2, we describe the experimental setup. We explain the engine dynamics in Section 3 and the approximation we 
made to obtain a linear equation in Section 4 . We then recall the notion of residue in Section 5. In Section 6, we describe our observer design. We conclude in Section 7 by conclusions and the future work

\section{EXPERIMENTAL SETUP FOR CONTROL DESIGN}

The synthesis of the state based observer-controller was first validated on a 4 cylinder DI engine model. This reference model is based on the Chmela combustion modelling (Chmela and Orthaber, 1999). The reference model included dynamics of the combustion and the transformation into indicated torque and after into effective torque. The transmission is represented as in (Chmela and Orthaber, 1999). A more realistic model with non linearities and effects due to the imperfection of flywheel tooth will be integrated in a second time. The observer has been tested with instantaneous engine speed measurements coming from the reference model and also tested on the test bench.

The synopsis of the complete simulation is described on the figure below. We implemented the simulation model (reference model + observer + controller) on a real time simulator based on xPCTarget (Support, n.d.). The real time ratio is not achieved due to the complexity of the reference model. With a $1 \mathrm{GHz}$ Pentium based computer, 1 second of engine simulation is computed in 30 seconds. This HiL platform was transferred to a fast prototyping system by adding specific input/ouputs boards. The same code was kept and implemented in the control system in order to be tested on the test bench.

\section{ENGINE DYNAMICS}

We want to reconstruct the combustion torque with crankshaft velocity as only measurement. Following (Kiencke and Nielsen, 2000) we write the dynamics equation. The torque balance on the crankshaft is given by

$$
\frac{d}{d \alpha}\left(\frac{1}{2} J(\alpha) \dot{\alpha}^{2}\right)=T_{c o m b}(\alpha)-T_{l o a d}(\alpha)-T_{\text {fric }}(\alpha)
$$

Let $T_{\text {mass }}=T_{\text {comb }}-T_{\text {load }}-T_{\text {fric }}$ denote the mass torque. The combustion torque is also referred to as indicated torque. We consider that the load torque $T_{\text {load }}$ is a low frequency signal and that friction torque is a second order polynomial in $\dot{\alpha}$ (Cavina and Ponti, 1999). We can split the mass torque into two terms

$$
T_{\text {mass }}=J(\alpha) \ddot{\alpha}+\frac{1}{2} \frac{d J}{d \alpha} \dot{\alpha}^{2}
$$

The first term represents the oscillating masses and the second one represents the rotating masses. We use a model of two massed connected by a single rod (Fehrenbach, 1990) (see also (Kiencke and Nielsen, 2000)). The overall rod mass $m_{\text {rod }}$ is

- an oscillating part $m_{\text {rod,osc }}=m_{\text {rod }} \frac{l_{o s c}}{l}$

- a rotating part $m_{\text {rod,rot }}=m_{\text {rod }} \frac{l_{\text {rot }}}{l}$

The two lengths $l_{\text {rot }}$ and $l_{\text {osc }}$ with $l_{\text {rot }}+l_{\text {osc }}=l$ are defined by the position of the center of gravity of the connecting rod. The oscillating mass is :

$$
m_{\text {osc }}=m_{\text {piston }}+m_{\text {rod }} \frac{l_{\text {osc }}}{l}
$$

and the rotational mass on each cylinder is:

$$
\frac{m_{\text {rot }}}{C Y L}=\frac{m_{\text {crank }}}{C Y L}+m_{\text {rod }} \frac{l_{\text {rot }}}{l}
$$

The crankshaft mass is deduced from the moment of inertia

$$
m_{\text {crank }}=\frac{J_{\text {Crank }}}{r^{2}}
$$

After further calculations we have

$$
T_{m a s s}=J \ddot{\alpha}+\frac{1}{2} \frac{d J}{d \alpha} \dot{\alpha}^{2}
$$

with

$$
\begin{aligned}
& \text { - } J(\alpha)=m_{\text {rot }} r^{2}+m_{o s c} \sum_{j=1}^{C Y L}\left(\frac{d s_{j}}{d \alpha}\right)^{2} \\
& \text { - } \frac{d J}{d \alpha}=2 m_{o s c} \sum_{j=1}^{C Y L} \frac{d s_{j}}{d \alpha} \frac{d^{2} s_{j}}{d \alpha^{2}}
\end{aligned}
$$

\section{NOTION OF RESIDUES AND USE FOR TORQUE BALANCING}

The residue is the relative error of the effective work. It is a classical notion for torque balancing as explained in (Kiencke and Nielsen, 2000). It can be written

$$
R_{i}=\frac{\frac{C Y L}{4 \pi} \int_{t=\alpha_{i}-\frac{2 \pi}{C Y L}}^{\alpha_{i}+\frac{2 \pi}{C Y L}} T_{i n d} d t-\frac{1}{4 \pi} \int_{t=0}^{4 \pi} T_{i n d} d t}{\frac{1}{4 \pi} \int_{t=0}^{4 \pi} T_{i n d} d t}
$$

where the angle on the crankshaft $\alpha_{i}$ represents the middle point between the TDC and the BDC of the cylinder $i$. By definition

$$
\sum_{i=1}^{C Y L} R_{i}=0
$$

The residues will allow us to know the equilibrium of the stroke. When the system is perfectly balanced the residues are 0 . A positive residue in cylinder $i$ reveals that this cylinder generates more torque than the average of the 4 cylinders. This unbalance can be corrected by injecting less fuel into cylinder $i$. If convergence time is not an issue, a simple integral controller can be used to correct cylinder $i$. More sophisticated adaptation laws can be developed and seem efficient in practice $($ see $(?))$ 


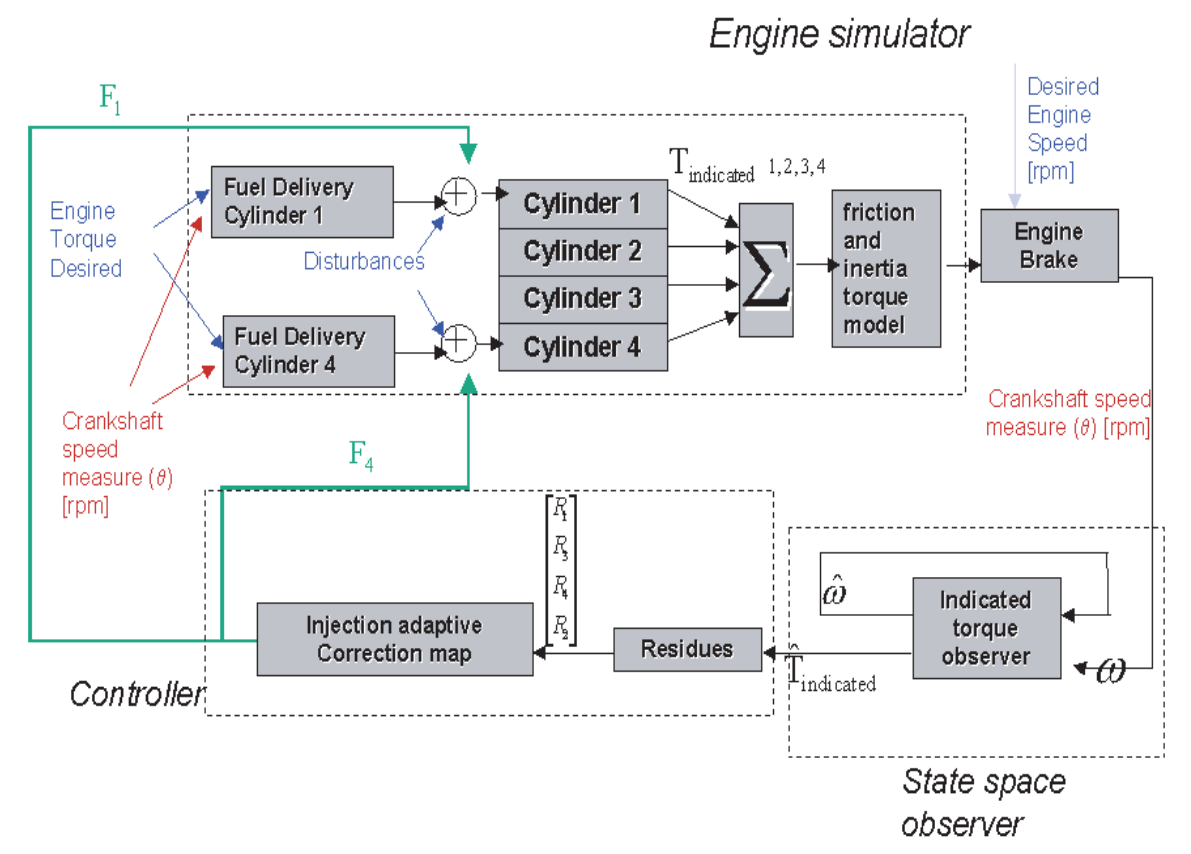

Figure 1. Global Scheme

\section{DISCRETE TIME-VARYING LINEAR APPROXIMATION}

For further calculations, the torque balance is an angle-dependent differential equation with timederivatives

$$
J(\alpha) \ddot{\alpha}=T_{\text {comb }}(\alpha)-T_{\text {load }}^{*}(\alpha)-f(\alpha) \dot{\alpha}^{2}
$$

with

$$
\text { - } f=\frac{d J}{d \alpha}
$$

- $T_{\text {load }}^{*}=T_{\text {load }}+T_{\text {fric }}$

$T_{\text {load }}^{*}$ refers to the extended torque. We can reformulate the second derivative of the crankshaft angle to have

$$
\dot{\alpha} \frac{d \dot{\alpha}}{d \alpha}=\frac{1}{J(\alpha)}\left(T_{c o m b}(\alpha)-T_{\text {load }}^{*}(\alpha)-f(\alpha) \dot{\alpha}^{2}\right)
$$

Using a first order approximation on the integration of the previous equation, we can break the dependence on time and on crankshaft angle and only save a dependence on the square of the crankshaft angle speed.

$$
\begin{aligned}
\dot{\alpha}^{2}(n+1) & -\dot{\alpha}^{2}(n) \approx \\
& \frac{2 \Delta \alpha}{J(n)}\left(T_{\text {comb }}(n)-T_{\text {load }}^{*}(n)-f(n) \dot{\alpha}^{2}(n)\right)
\end{aligned}
$$

We use as angular path $\Delta \alpha=6^{\circ}$. The model is then discretized with respect to angle steps instead of time steps. Using the square of the crankshaft angle speed $\dot{\alpha}^{2}$ as the first state variable $x_{1}$, we get the linear equation

$$
x_{1}(n+1)=\left(1-\frac{2 \Delta \alpha}{J(n)} f(n)\right) x_{1}(n)+\frac{2 \Delta \alpha}{J(n)} x_{2}(n)
$$

where

$$
\left\{\begin{array}{l}
x_{1}(n)=\dot{\alpha}^{2}(n) \\
x_{2}(n)=T_{\text {comb }}(n)-T_{\text {load }}^{*}(n)
\end{array}\right.
$$

This formulation of the problem will allow us to use classical methods of control to estimate the indicated torque.

\section{OBSERVATION OF THE INDICATED TORQUE}

From the previous dynamics we look for an observer of the system to estimate the indicated torque. The system has a two-dimensional state and a single measurement (crankshaft speed). It must be complemented with another equation reflecting the evolution of $T_{\text {comb }}(n)-T_{\text {load }}^{*}(n)$.

\section{$6.1 x_{2}$ considered as an unknown constant}

As a first try we consider that the variation of the indicated torque during one angular step is small.

6.1.1. Observation Model We use as second equation

$$
\frac{d x_{2}}{d \alpha}=0
$$


during $\Delta \alpha$. Between two angular steps we get

$$
\left\{\begin{array}{c}
x_{1}(n+1)=\left(1-\frac{2 \Delta \alpha}{J(n)} f(n)\right) x_{1}(n)+\frac{2 \Delta \alpha}{J(n)} x_{2}(n) \\
x_{2}(n+1)=x_{2}(n)
\end{array}\right.
$$

We have the system

$$
\left\{\begin{array}{c}
X(n+1)=A(n) X(n) \\
y(n)=C X(n)
\end{array}\right.
$$

with

$$
\begin{aligned}
& \text { - } X(n)=\left[\begin{array}{ll}
\dot{\alpha}^{2}(n) & T_{\text {comb }}(n)-T_{\text {load }}^{*}(n)
\end{array}\right]^{T} \\
& \text { - } A(n)=\left[\begin{array}{cc}
\left(1-\frac{2 \Delta \alpha}{J(n)} f(n)\right) & \frac{2 \Delta \alpha}{J(n)} \\
0 & 1
\end{array}\right] \\
& \text { - } C=\left[\begin{array}{ll}
1 & 0
\end{array}\right]
\end{aligned}
$$

We build the observer by linear feedback on the crankshaft angular speed.

$$
\left\{\begin{aligned}
\hat{X}(n+1) & =A(n) \hat{X}(n)-L(n)(\hat{y}(n)-y(n)) \\
\hat{y}(n) & =C \hat{X}(n)
\end{aligned}\right.
$$

so the error satisfies

$$
\left\{\begin{aligned}
\tilde{X}(n+1) & =(A(n)-L(n) C) \tilde{X}(n) \\
\tilde{y}(n) & =C \tilde{X}(n)
\end{aligned}\right.
$$

with $\tilde{X}(n)=X(n)-\hat{X}(n)$.

6.1.2. Observer convergence The dynamics of our system is periodic, so we just have to check stability for $\prod_{k=1}^{T}\left(A_{k}-L_{k} C\right)$. We numerically investigate the convergence of the system. The eigenvalues of $\prod_{k=1}^{T}\left(A_{k}-L_{k} C\right)$ are $\left[0,10^{-4}\right]^{T}$ which proves the convergence of the observer.

\subsubsection{Results and Comments}

6.1.3.1. Simulation results In Figures 2, 3, and 4 we have

- ref : reference

- pp1 : result of the first observer with pole placement $\frac{d x_{2}}{d \alpha}=0$

The simulation is at 1000rpm with an BMEP of $2 \mathrm{bar}$. For the simulation part, we introduced offsets on the mass injected in each cylinder.

- Cylinder 1: $20 \%$ of the reference mass

- Cylinder 2: $0 \%$ of the reference mass

- Cylinder 3: $-20 \%$ of the reference mass

- Cylinder 4: $5 \%$ of the reference mass

We introduced also some noise for the simulation which reproduces the imperfection of the flywheel.

6.1.3.2. Results from the bench Figures 5 and 6 display the result of the estimator on experimental data. We reconstruct the indicated torque from

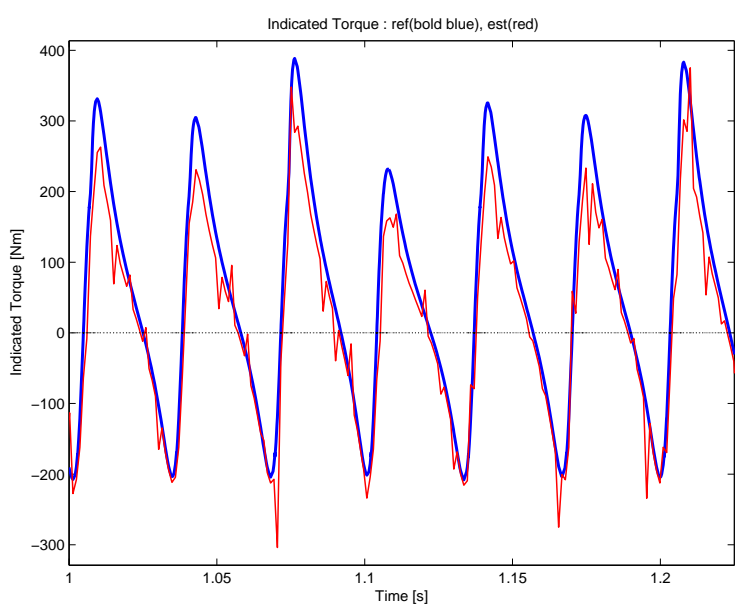

Figure 2. Indicated torque (1000rpm, 2bar)
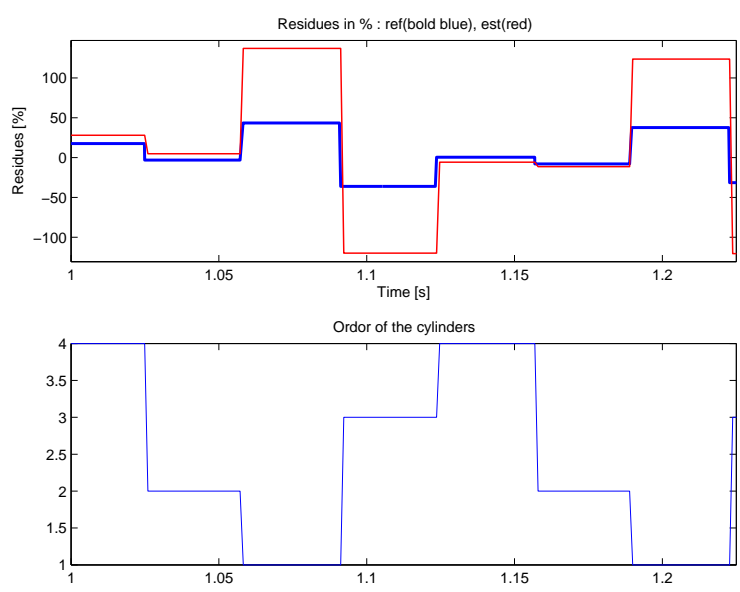

Figure 3. Residues (1000rpm, 2bar)

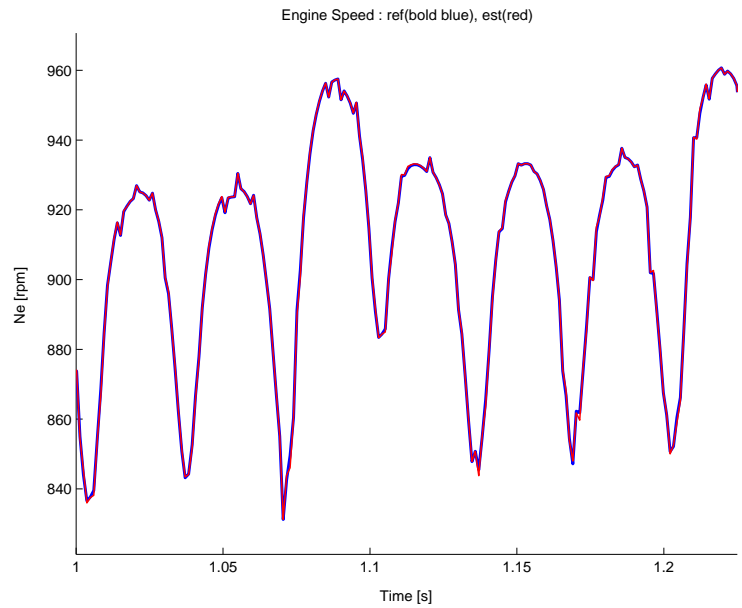

Figure 4. Engine Speed (1000rpm, 2bar)

the bench with the in-cylinder pressure and we test the observer on the flywheel velocity measurement. The setting point is not the same as the simulation one.

6.1.3.3. Comments The simulation and the result on the bench are relatively satisfactory. Qualitatively, the residues are well estimated. On the 


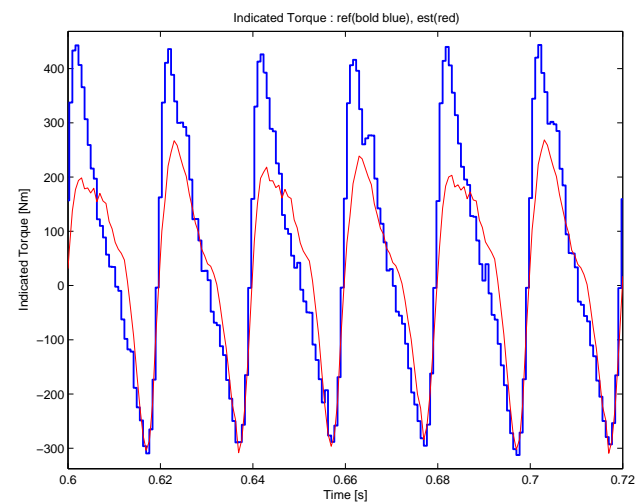

Figure 5. Indicated torque on the bench
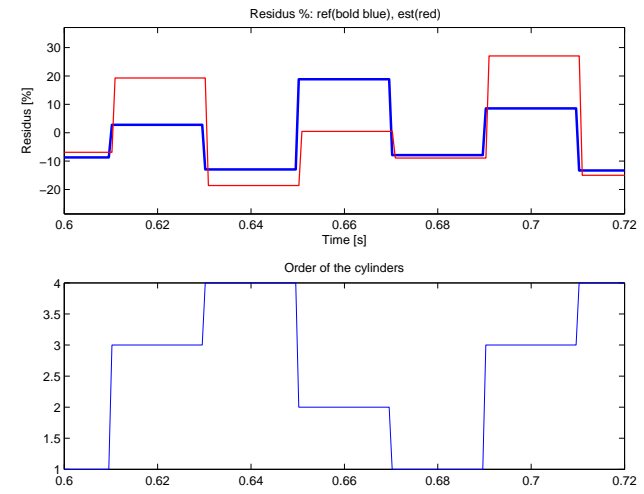

Figure 6. Residues on the bench

other hand the quantitative prediction is not accurate. In order to drive the residues to zeros, only the qualitative information matters: simple PID would work on this fully actuated system provided the sign of the gains are known. The idea behind this observer is the simplest as we introduced no more knowledge about the system. Using more physics on the second equation, we now aim at increasing the quality of the observation.

$6.2 x_{2}$ modelled as a stable second order filter with known parameters

6.2.1. Observation Model The second observer is based on the fact that the indicated torque is periodic, so we consider $x_{2}$ as a stable second order filter with known parameters. We use for second equation

$$
x_{2}(n)=a(n)+b(n) \sin (n \Delta \alpha)
$$

As the indicated torque varies in transient mode (according to torque and engine speed), the parameters $a$ and $b$ must be adapted. Both $a$ and $b$ are now included in the state. The dynamics reads

$$
\left\{\begin{array}{c}
X(n+1)=A(n) X(n) \\
y(n)=C X(n)
\end{array}\right.
$$

with

$$
\text { - } X(n)=\left[\begin{array}{lll}
\dot{\alpha}^{2}(n) & a(n) b(n)
\end{array}\right]^{T}
$$

$$
\begin{aligned}
& \text { - } A(n)=\left[\begin{array}{cccc}
\left(1-\frac{2 \Delta \alpha}{J(n)} f(n)\right) & \frac{2 \Delta \alpha}{J(n)} & \frac{2 \Delta \alpha}{J(n)} \sin (n \Delta \alpha) \\
0 & 1 & 0 \\
0 & 0 & 1
\end{array}\right] \\
& \text { - } C=\left[\begin{array}{lll}
1 & 0 & 0
\end{array}\right]
\end{aligned}
$$

Again, we build the predictor by linear feedback on the crankshaft angular speed. The observer dynamics reads

$$
\left\{\begin{aligned}
\hat{X}(n+1) & =A(n) \hat{X}(n)-L(n)(\hat{y}(n)-y(n)) \\
\hat{y}(n) & =C \hat{X}(n)
\end{aligned}\right.
$$

so the error satisfies

$$
\left\{\begin{aligned}
\tilde{X}(n+1) & =(A(n)-L(n) C) \tilde{X}(n) \\
\tilde{y}(n) & =C \tilde{X}(n)
\end{aligned}\right.
$$

with $\tilde{X}(n)=X(n)-\hat{X}(n)$.

6.2.2. Observer convergence As we said above, the dynamics is periodic so we just have to check stability for $\prod_{k=1}^{T}\left(A_{k}-L_{k} C\right)$. The eigenvalues of $\prod_{k=1}^{T}\left(A_{k}-L_{k} C\right)$ are $\left[0.67,-3.510^{-17}, 5.210^{-4}\right]^{T}$ which proves the convergence of the observer. These last eigenvalues correspond to an average model $\bar{A}$ with eigenvalues having the following modulus $[0.9933,0.5318,0.8816]^{T}$. A question of particular interest is the design of the gain scheduling that would give an improved convergence. This is a point we are focusing on.

\subsubsection{Results and Comments}

6.2.3.1. Results from the simulation Figures 7 and 8 show the comparaison of the two observers and the better results of the second one.

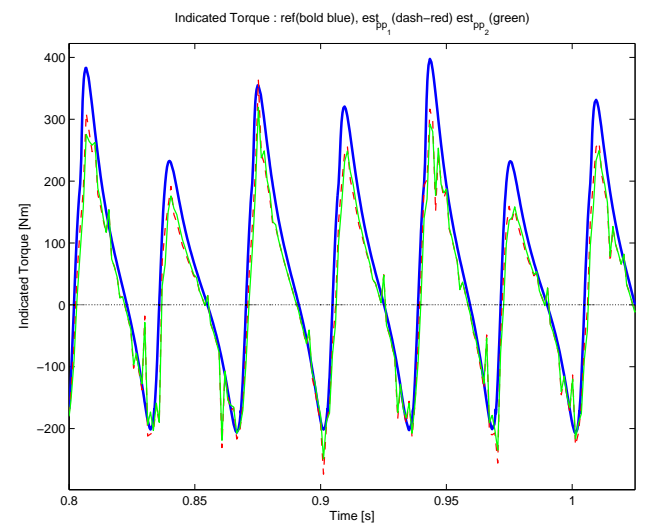

Figure 7. Indicated torque with the second observer

6.2.3.2. Comments Accuracy is improved but remains an issue. Convergence is obtained in practice but its interpretation is tricky. Indeed, for a given $n$, the pair $(A(n), C)$ is not observable. One eigenvalue of the observability matrix is always 0 . 

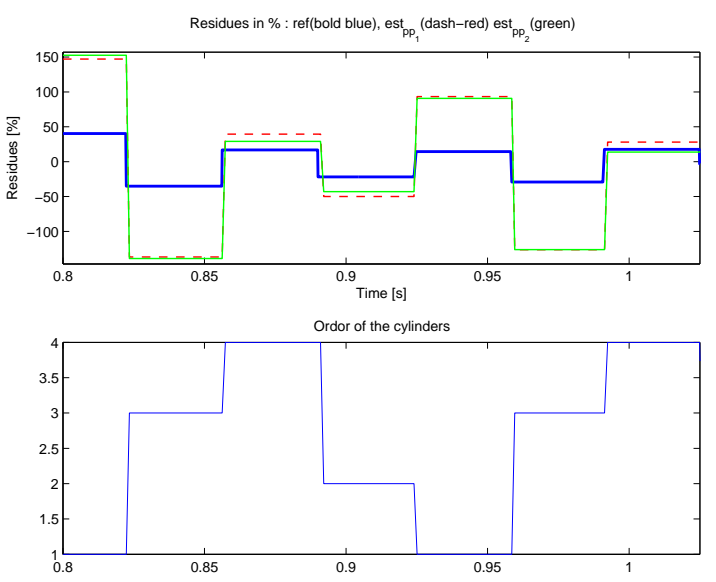

Figure 8. Residues with the second observer

Thus the gain scheduling design is somewhat more difficult to achieve. Today it seems this formulation of the problem could be improved further, by adding more harmonics to the approximation for instance.

\section{CONCLUSION AND FUTURE WORK}

This article summarizes the evolution of the observer design at IFP for torque balancing on DI engine. After explaining the engine dynamics in Section 3 and the model we use in Section 4, we explored two ways to describe the evolution of the indicated torque and showed the results on simulation and on the bench (Section 6).

So far we have some good results on the estimation of the torque balance, though their quantitative accuracy can be improved. By using the periodicity of the indicated torque and a first harmonic development, we have better results but have not finished the exploration of this method yet. The quality of the observation was improved and a proof of the convergence of this type of observer is in progress.

Future directions seem clear. For the control design method, we have to evaluate the strengths and weaknesses of the previous methods by increasing the size of the decomposition (by adding terms in $\cos (\alpha), \sin (2 \alpha), \cos (2 \alpha), \ldots)$. Concerning physical modelling, an other estimation of the load torque, not depending on the single time estimation of the indicated torque at TDC and BDC. We will have to introduce a more accurate model of transmission and its non linearities. This part will be required for use on a vehicle whose transmission is less stiff. Moreover, the problem of torque balancing can be included in the more global problem of cylinder balancing which include torque balancing but also air fuel ratio regulation.

\section{NOMENCLATURE}

\begin{tabular}{|c|c|c|}
\hline Symbol & Units & Physical Variables \\
\hline BMEP & [bar] & Break Mean Effective Pressure \\
\hline BTC & & Bottom-Top Center \\
\hline CYL & & Number of cylinders \\
\hline$J$ & {$\left[\mathrm{kgm}^{2}\right]$} & Moment of inertia \\
\hline$J_{\text {crank }}$ & {$\left[\mathrm{kgm}^{2}\right]$} & Crankshaft moment of inertia \\
\hline 1 & {$[\mathrm{~m}]$} & Connecting rod lenght \\
\hline$m_{\text {crank }}$ & {$[\mathrm{kg}]$} & Crankshaft mass \\
\hline$m_{o s c}$ & [kg] & Oscillating mass \\
\hline$m_{\text {rod }}$ & [kg] & Rod mass \\
\hline$m_{\text {rod,osc }}$ & [kg] & Oscillating rod mass \\
\hline$m_{\text {rod,rot }}$ & [kg] & Rotational rod mass \\
\hline $\mathrm{r}$ & {$[\mathrm{m}]$} & Radius of the crankshaft \\
\hline$R_{i}$ & & Residue of cylinder $i$ \\
\hline$s$ & [m] & Piston stroke \\
\hline$\dot{s}$ & {$[\mathrm{~m} / \mathrm{s}]$} & Piston velocity \\
\hline$s_{j}$ & {$[\mathrm{~m}]$} & Piston velocity of cylinder $j$ \\
\hline TDC & & Top-Down Center \\
\hline$T_{c o m b}$ & {$[\mathrm{Nm}]$} & Combustion torque \\
\hline$T_{\text {fric }}$ & {$[\mathrm{Nm}]$} & Friction torque \\
\hline$T_{\text {ind }}$ & {$[\mathrm{Nm}]$} & Indicated torque \\
\hline$T_{\text {load }}$ & {$[\mathrm{Nm}]$} & Load torque \\
\hline$T_{\text {load }}^{*}$ & {$[\mathrm{Nm}]$} & Extended load torque \\
\hline
\end{tabular}

\section{REFERENCES}

Cavina, N. and F. Ponti (1999). Fast algorithm for on-board torque estimation. In: Proc. of SAE Conference.

Chmela, F. and G. Orthaber (1999). Rate of heat release prediction for direct injection diesel engines based on purely mixing controlled combustion. In: Proc. of SAE Conference. number 1999-01-0186.

Fehrenbach, H. (1990). Model-based combustion pressure computation through crankshaft angular accelerartion analysis. Proceedings of $22^{\text {nd }}$ International Symposium on Automotive Technology.

Ginoux, S. and J.C. Champoussin (1997). Engine torque determination by crankangle measurments: State of art, future prospects. In: Proc. of SAE Conference. number 970532.

Jianqiu, L., Y. Minggao, Z. Ming and L. Xihao (2002a). Advanced torque estimation and control algorithm of diesel engines. In: Proc. of SAE Conference.

Jianqiu, L., Y. Minggao, Z. Ming and L. Xihao (2002b). Individual cylinder control of diesel engines. In: Proc. of SAE Conference. number 2002-01-0199.

Kiencke, U. and L. Nielsen (2000). Automotive Control Systems For Engine, Driveline, and Vehicle. SAE Internationnal.

Support, Mathworks XPC Target 2 (n.d.). http://www.mathworks.com/products/xpctarget.

van Nieuwstadt, M.J. and I. Kolmanovsky (1997). Cylinder balancing of direct injection engines. In: Proc. of the IEEE Conf. American Control Conference. 